\title{
CONTRIBUIÇÃO PARA O CONHECIMENTO DA FLORA ALGOLÓGICA MARINHA DO ESTADO DO PARANÁ
}

(Trabalho apresentado na 2. ${ }^{a}$ Reunião Anual da Sociedade Botânica do Brasil, em Viçosa - Janeiro de 1951).

I - Zonação e distribuição segundo habitats.

Aylthon Brandão Joly (*)

\section{INTRODUÇÃO}

A presente nota, pretende dar uma idéia de como se distribuem várias espécies de algas, tanto por diferentes habitats (seja rochoso ou limoso) como também a relação que mantêm entre si, nos rochedos à beira mar.

Para maior facilidade de exposição, grupâmo-las de acôrdo com o caráter de litoral onde vivem. Relacionaremos primeiro as que se encontram nos "costões" rochosos expostos à ação violenta da arrebentação. Em segundo lugar, as que habitam as costas rochosas também, mas protegidas da ação intensa das ondas, seja a costa interior de uma ilha muito próxima do continente, ou seja uma das faces de pequenos cabos ou penínsulas que, por determinadas cireunstâncias geográficas se encontrem abrigados. Em terceiro, mencionaremos a costa brejosa formada no estado do Paraná por manguesais, do tipo já conhecido entre nós, desde os trabalhos de Luederwaldt (6) e Rawitscher (9).

Além dêstes 3 tipos de habitats, que são fàcilmente accessíveis, será necessário considerar ainda os habitats de algas de maior profundidade,

(*) Departamento de Botânica - Faculdade de Filosofia, Ciências e Letras da Universidade de São Paulo.

Queremos agradecer aqui, a orientação e colaboração dos Profs. F. Rawitscher e Wm. R. Taylor que despertaram no autor, o interêsse por êste grupo e ao Prof. E. Simões de Paula, Diretor da Faculdade de Filosofia, Ciêneias e Letras da Universidade de São Paulo, pelas facilidades materiais proporeionadas para as excursões e aparelhamentos. 
dos quais só possuimos conhecimentos esporádicos, por absoluta falta de dragagens que pudessem fornecer material para um conhecimento da nossa flora da região sub-litorânea.

A região em questão, foi detalhadamente pesquizada do ponto de vista geológico-geográfico em recente trabalho de Bigarella (1) do qual emprestamos um dos mapas que ilustram a presente nota. Tôdas as referências a rochas da costa baseiam-se também no mencionado trabalho.

A costa paranaense não está sujeita a grandes desníveis de maré, salvo por ocasião de marés especiais; geralmente os desníveis diários não vão além de $0,90 \mathrm{~m}$. a $1,00 \mathrm{~m}$. (cf. publ. mensal Cia. Mate Laranjeiras Tabelas de marés - Pôrto de Paranaguá).

Infelizmente não existem dados sôbre a variação da temperatura da água do mar durante os meses do ano, assim como faltam também dados sôbre a salinidade e turbidez, dados êsses que sem dúvida poderiam precisar as condições ecológicas reinantes.

Muitas das espécies aqui mencionadas foram identificadas pelo autor, baseando-se em comparações com material já determinado pelo Prof. Wm. Taylor da Universidade de Michigan. Outras algas relacionadas aparecem só com o nome genérico; tal se deve à impossibilidade do autor de fazer identificações específicas, que, sem dúvida, caracterizariam melhor a flora descrita, pela falta de exemplares autênticos para comparação e de bibliografia especializada. Nestas identificações genéricas foram utilizados os trabalhos de Taylor (13), Fritsch (5), Engler e Prantl (4) e De Toni (3).

Esta pequena nota está longe de ser uma flora algológica do litoral paranaense, do qual o autor pereorreu uma pequena parte, mas pretende com ela iniciar uma série de publicações, que venham pouco a pouco se completando, para talvez, mais tarde, tentarmos uma publicação que reúna, ao lado de ilustrações e descrições das espécies, dados biológicos e ecológicos. (*)

(*) Os únicos trabalhos existentes em português, que trazem listas de algas são os de Rawitscher (9), e Luetzelburg (7). Luederwaldt (6) meneiona três algas para o manguesal de Santos; duas delas estão determinadas como: Bostrychia brasiliana e Catenella impudica; provàvelmente trata-se das que hoje denominamos de $B$. radicans e C. repens. Oliveira (16), trás poucos gêneros de algas para a baía de Guanabara. 


\section{1) DISTRIBUIÇÃO DE ALGAS NAS COSTAS ROCHOSAS SU- JEITAS A FORTE ARREBENTAÇ̃̃O DO LITORAL PARA- NAENSE (CAIOBÁ)}

A fig. 1 nos mostra o litoral paranaense, vendo-se assinaladas as regiões de Caiobá, Matinho e Passagem, de onde provêm as algas aqui referidas; tem uma extensão total aproximada de $90 \mathrm{Km}$ (ef. 10 p. 207). Pràticamente todo o litoral interior das grandes baías de Paranaguá e Guaratuba é formado por manguesais; as praias arenosas são muito extensas também (cf. 1 fig. 1 e 10 p. 207-208), de modo que o litoral rochoso especialmente favorável ao desenvolvimento de algas é bastante limitado nas costas paranaenses.

Para dar uma idéia da extensão arenosa das praias, basta mencionarmos que duas delas, a praia Deserta $(36 \mathrm{Km})$ e a praia de Leste com quase $30 \mathrm{Km}$, perfazem mais de dois têrȩos de todo o litoral (cf. 10 p. 207-208).

A figura seguinte apresenta um esquema de rochas, que se encontram no fim da praia de Caiobá, ao lado do morro dêsse nome (ef. 1 fig. 7), pertencentes ao Complexo Cristalino Brasileiro e que servem de substrato para a flora algológica aqui deserita. Aparecem numerados para facilitar a compreensão do texto, os diferentes tipos de habitats de algas.

Tipo 1 - Representado pela parte superior das rochas, que normalmente nunca é coberta por água do mar, mas só "lavada" pelas ondas mais fortes durante a maré cheia, e por água de chuva. Zona Phycologicamente pobre, habitada especialmente por uma Cyanophycea, Lyngbya confervoides, que forma extensas placas verde-oliva escuras e fortemente aderidas à rocha. Estas, quando umedecidas, tornam-se extremamente escorregadias e perigosas para o eaminhar sôbre as pedras. Esta zona está sujeita a grandes variações térmicas diárias, devido à insolação direta durante todo o dia.

Tipo 2 - Êste habitat, entre os limites da maré alta e da maré baixa normais corresponde à "Gezeitenzone" dos autores alemães ou à "inter-tidal zone" dos autores de língua inglesa. É perfeitamente delimitado na sua parte superior pela presença de uma faixa, de largura mais ou menos uniforme de Balanus sp. (craca) acima, e um pouco de Mytilus perna (marisco); é um dos mais ricos em algas tanto pelo número como pela variedade de indivíduos. Esta zona, que periòdicamente fica submersa ou exposta, é a que mais está sujeita à ação violenta da "arrebentação" (Ressaca). Possue, como é de se esperar, uma flora típica e especial. Em Caiobá, aparece no limite superior desta faixa, uma Phaeophycea, Levringia sp. quase 
que dominante nesta parte mais alta, seguida um pouco mais abaixo por Cladophora fascicularis, Chaetomorpha media e Ulva fasciata, (neste habitat representado por formas com talos alongados de margens crespas e estreitas, formando, cada indivíduo, tufos mais ou menos isolados). Muito frequente nesta região é a Centroceras clavulatum pequena Rhodophycea, e também formando tufos, Chnoospora pacifica e a calcárea Jania rubens. Estas seis espécies associadas a um Ectocarpus que cresce especialmente sôbre Mytitlus perna, são as mais características e frequentes do litoral paranaense, em Caiobá. Ao lado destas encontram-se também outras Rhodophyceas, como por exemplo a grande Grateloupia cuneifolia, de côr verde, lembrando mais uma Ulva do que uma alga vermelha e Grateloupia sp; nas partes mais baixas desta zona já aparecem os tufos de Hypnea musciformis, associada a Pterosiphonia pennata e a Bryocladia thyrsigera. Estas duas especialmente, são as dominantes na parte mais baixa das rochas onde estas mergulham na areia. Afora estas algas vistosas, características e abundantes, pudemos ainda constatar a presença de mais algumas mencionadas a seguir: Cladophora sp. raros exemplares aparentemente jovens, sem a forma típica, indetermináveis. Sargassum sp., indivíduos jovens não muito frequentes. Duas pequeninas epífitas erescendo especialmente sôbre Chaetomorpha media: Erytrocladia subintegra e Erytrotrychia sp. Estas só podem ser observadas com auxílio de uma boa lupa ou microscópio. Pode-se notar, no entretanto, nas extremidades dos filamentos de $\mathrm{Ch}$. media, especialmente depois da liberação de zoósporos, pequenos pontos avermelhados, mais ou menos reunidos ou espalhados, denotando a existêneia destas colônias de epífitas.

\section{Limites entre as zonas 1 e 2}

Na parte mais alta da zona 2, encoberta sòmente poucas horas por dia pela maré alta, encontramos nesta época, (novembro 1950), exclusivamente algas do gênero Porphyra, que aí tem seu habitat favorável e atingem grande desenvolvimento, formando talos ora alongados ora expandidos, podendo atingir até $15 \mathrm{~cm}$. ou mais de largura, de uma bonita côr verde claro ou vermelho roxo. Neste habitat encontram-se também pequenos ou jovens (?) de Balanus sp. (a conhecida "craca" pequena) que marea assim, mesmo visto ao longe, o limite diário da água salgada nas rochas.

Tipo 3 - Representado pelas pequenas fendas e buracos que podem ser periòdicamente enchidos por água do mar ou das chuvas. 
Nestes buracos temos provàvelmente grandes variações de salinidade, por causa da diluição pelas chuvas e da evaporação que condensa a solução. Só algumas algas (as "Euryhalinas" de Oltmanns ' 8 ') conseguem viver em tais condições. Há, também, grande variação de temperatura, pois a água aqui retida é fortemente aquecida durante o dia, mas assim mesmo pode manter uma vegetação (algas "Euritérmicas"' seg. Oltmanns ' 8 ') já relativamente rica, sobretudo nas poças e fendas mais fundas. Assim é que aqui encontramos algas que se desenvolvem melhor nos limites entre as zonas 1 e 3 e também as do tipo 4 como vamos ver. Pudemos anotar as seguintes com mais frequência encontradas: Ulva fasciata, Gymnogongrus Griffithsiae, Porphyra sp. e Ceramium sp. Tôdas pequenas e mal formadas.

Tipo 4 - Êste habitat, representado em Caiobá por pequenas depressões entre fragmentos de rochas, geralmente bem ao lado ou por trás de um anteparo rochoso maior, como mostra o esquema (fig. 2), também possue sua flora mais ou menos característica. São nestas poças, onde a água é constantemente renovada, que se encontram os melhores exemplares de Ulva fasciata, aqui bem desenvolvidos pois não sofrem a ação da arrebentação violenta que earacteriza o habitat $n,{ }^{\circ} 2$. Aparecem duas outras algas vermelhas, formando verdadeiras almofadas, tão denso é o seu crescimento: Gymnogongrus Griffithsiae e Amphiroa Beauvoisii. Podem também ser encontrados nos lados verticais dos primeiros blocos de pedra sujeitos à "Ressaca", voltando para o mar, exemplares de Centroceras clavulatum e uma ou outra planta de Chaetomorpha media ou Chnoospora pacifica. São frequentes também exemplares de Ulva fasciata e Porphyra sp. bem jovens e que iniciam seu desenvolvimento, revestindo quase tôdas as pequenas pedras ou pedaços de conchas que por aí se encontrem. É interessante assinalar, que os exemplares adultos de Porphyra sp., estão colocados bem mais acima, nos limites entre os habitats 1 e 2 .

Tipo 5 - Não representado no esquema, é o habitat que poderíamos designar como o das Enteromorpha. Está localizado pouco abaixo do limite superior da maré alta, na face voltada para a praia, nos rochedos acima esquematizados. As algas encontram-se erescendo em grandes massas filamentosas, fixas à rocha no limite destas com a areia, de modo a ficarem, durante a maré baixa mais ou menos enterradas, ou pelo menos com muita areia sempre úmida entre os fios. Em Caiobá encontram-se, pelo menos, 3 espécies de Enteromorpha, E. Linza, $E$. lingulata e $E$. flexuosa, associadas a Rhizoclonium riparium 
e Chaetomorpha brachygona. Estas Enteromorphas apresentam-se sempre com boa parte da extremidade dos fios, braneos e murehos, denotando a liberação de zoósporos. É especialmente notável pelo tamanho e largura das frondes verdes. $E$. Linza, podendo atingir até $2 \mathrm{~cm}$. de largura e de um bonito verde claro. Estas algas aqui assinaladas, frequentemente estão sujeitas à ação de água doce de pequenas nascentes e riachos, que em geral saem no mar junto de encostas rochosas como a do tipo descrito. Esta Influência deve ser particularmente notada durante as horas de baixa-mar quando a água doce pode prevalecer.

Denominação e caracterização phycológica dos habitats acima descritos

- Propomos para a caracterização do tipo $\mathrm{n}^{0}{ }^{0}$, a designação de zona da Lyngbya por ser esta a única alga que aí se encontra. $\quad$ interessante acrescentar que nesta zona vivem pequenos moluseos do gênero Littorina (pelo menos $2 \mathrm{spp}$.) que também earacterizam, juntamente com a alga mencionada êste habitat.

O segundo tipo, o mais rico e variado em espécies e indivíduos, é também a zona característica dêste tipo de litoral no Estado do Paraná e para ela propomos a designação de zona Levringia-ChaetomorphaCentroceras. Seguem-se depois as outras algas mencionadas anteriormente. Também aqui será necessário acrescentar a presença de Balanus e Mytilus (craca e mariseo) tão earacterísticos das nossas pedras do litoral. (**)

O terceiro tipo, que depende muito das condições locais dos rochedos, poderíamos designar como habitat de Ulva-Gymnogongrus.

O quarto tipo será o habitat da Clva-Amphiroa-Gymnogongrus, que são as mais características.

O quinto tipo é o habitat das Enteromorpha e Rhizoclonium.

(*) Reservamos a designação de zona para os habitats 1 e 2 . Os outros habitats (3,4 e 5) são apenas variações locais, que dependem muito da topografia dos rochedos, e não constituem "zonas", na acep̧̧ão da palavra e na tradị̧ão phycoecológica.

$\left({ }^{* *}\right)$ Queremos assinalar aqui pela primeira vez, a identidade básica na distribuição das zonas de algas e animais marinhos, das nossas costas, rochosas com as da África do Sul - ef. Stephenson $(11,12)$ e também o resumo de Chapman (2) baseando-nos nos vários trabalhos de Stephenson e seus colaboradores. 
2) DISTRIBUIÇÃO DE ALGAS NAS COSTAS ROCHOSAS, MAIS CALMAS SEM ARREBENTAÇÃO FORTE NO LITORAL PARANAENSE (CAIOBÁ)

$\mathrm{O}$ ambiente a ser deserito, difere bastante do primeiro, essencialmente pela falta de movimentação violenta das ondas; é atualmente um ponto de sedimentação (ver fig. 3) onde se acumula muita areia entre os blocos soltos. Além disso, a transparência da água, especialmente durante a maré baixa é quase nula, tal a quantidade de material organo-argiloso mantido em suspensão, trazido dos manguesais próximos e aí acumulados.

Notamos uma modificação completa na vegetação quando comparamos o primeiro ambiente já descrito.

Podemos para êste ambiente descrever 3 tipos de habitats.

Tipo 1 - Esta Zona, descoberta totalmente durante a maré baixa, possue uma flora bem pobre. As pedras, em geral, apresentam-se revestidas com Lyngbya confervoides, o que as torna extremamente escorregadias, encontrando-se também, parcialmente cobertas com areia fina e limo. São frequentes os exemplares de Monostroma sp. Porphyra sp. (nas partes mais altas das rochas) e de duas espécies de Enteromorpha, E. lingulata e E. flexuosa. Aparece também aqui, nas poças maiores, uma espécie de Gelidium.

Tipo 2 - À medida que eaminhamos para o bordo marítimo dêste pequeno dique (à direita no esquema - fig. 3), que une a Ilha do Farol ao continente, notamos progressivamente um aumento da vegetação. Assim é que começam a aparecer exemplares de Callithamnion sp. ao lado da frequente Gigartina Teedii, que reveste totalmente os bordos dos blocos de basalto, bem como os densos tapetes formados por aglomerados de Laurencia papillosa e Caulerpa fastigiata (?). Encontram-se afora êstes, exemplares bem desenvolvidos de Gracilaria dominguensis e Pterocladia capillacea, misturados com tufos de Hypnea musciformis, Bryocladia thyrsigera, Bryothamnion Seaforthii, Pterosiphonia pennata e Bostrychia Binderi. Finalmente na parte inferior desta zona, aparece em abundância Sargassum cymosum e também a var. stenophyllum que atinge tamanho considerável (até $40 \mathrm{~cm}$., segundo exemplares colhidos em 4-11-1950). Sôbre êstes, crescem plantinhas de Plocamium sp. (provàvelmente $P$. brasiliensis) Ceramium sp. bem como emaranhados de Hypnea musciformis. Encontramos, também, exemplares de Padina Vickersiae, Codium decorticatum e Colpomenia sinuosa. Estas são as mais frequentes e vistosas. Poderemos mencionar ainda, raros exemplares de uma Cladophora sp. de côr verde escura com ramos duros, não bem 
desenvolvidos e um Ectocarpus sp. Ao afastarmo-nos dêsse dique de diabásio, agora em direção à terra, sôbre grandes matacões de gneis, que bordejam a praia, podemos encontrar na parte mais alta, atingida pela maré cheia, Bostrychia radicans e Gymnogongrus Griffithsiae e na zona entre as marés, Cladophora fascicularis muito ramificada e de côr verde claro, associada a Gracilaria dominguensis e Ulva fasciata e no limite inferior com Sargassum cymosum.

Tipo 3 - Esta zona da qual só temos notícias por meio de material recém atirado à praia próxima, parece possuir flora bem diferente daquela acima descrita. Tudo leva-nos a erer, tratarse de plantas de profundidade, que nunca são expostas pelas marés baixas normais. Assim pudemos anotar 3 espécies, pelo menos, impossíveis de eneontrar no estado adulto nas rochas descobertas. São as seguintes: Plocamium brasiliensis, Rhodymenia Palmetta e Spatoglossum Schroederi. Só com dragagens no local, êste habitat poderá ser conhecido.

Denominação e caracterização phycológica dos habitats acima descritos.

Propomos para o tipo 1 dêste habitat a designação de zona da Lyngbya-Monostroma-Enteromorpha.

O habitat n. 2 será conhecido como zona da Laurencia-GigartinaCallithamnion-Sargassum, que são os mais frequentes.

\section{ALGAS DOS MANGUESAIS E ADJACENCIAS NO LITORAL PARANAENSE (CAIOBÁ-PASSAGEM)}

As algas que habitam os manguesais são restritas a um pequeno grupo, quase tôdas de tamanho pequeno e com côres não vistosas.

Creseem sôbre qualquer galho de árvore, troncos semi-enterrados no lodo, bem como sôbre os pneumatóforos de Avicennia tomentosa ou as raizes suportes de Rhizophora mangle p. ex.

Pudemos anotar as seguintes, que são as mesmas por nós eneontradas nos manguesais de Santos, S. Sebastião e Peruibe no Estado de São Paulo: Bostrychia radicans, Caloglossa Leprieurii, Cladophoropsis membranacea, Lyngbya confervoides e Dermocarpa sp. que são as que dominam a associação: outras algas por nós coletadas, parecem preferir, no entanto, os bordos marítimos dêstes manguesais, como é o caso, por exemplo, de Monostroma sp., Bostrychia Binderi, Catenella repens (rara) e certas Enteromorpha (2 espécies pelo menos) já um pouco afastadas do manguesal pròpriamente dito e também Sirocoleum sp. 
É interessante assinalar que sôbre êstes fios de Enteromorpha cresce em abundância uma Diatomacea, Schizonema sp? formando fios gelatinosos que retêm os indivíduos; de mistura com êles encontram-se uma Cyanophycea, Stigonema sp. e uma Rhodophycea, cujo gênero é agora pela primeira vez assinalado na costa brasileira (*) Goniotrichum sp.

\section{LISTA PROVISÓRIA DAS ESPÉCIES}

MYXOPHYCEAE (Cyanophyceae)

Dermocarpa sp.

Lyngbya confervoides C. Ag. ex Gom.

Stigonema sp.

Sirocoleum sp.

\section{CHLOROPHYCEAE}

Enteromorpha flexuosa (Wulf.) J. Ag.

Enteromorpha Linza (L.) J. Ag.

Enteromorpha lingulata J. Ag.

Monostroma sp.

Ulva fasciata Delile

Chaetomorpha brachygona Harv.

Chaetomorpha media (C. Ag.) Kütz.

Cladophora sp.

Cladophora fascicularis (Mert.) Kütz.

Cladophoropsis membranacea (C. Ag.) Borg.

Rhizoclonium riparium (Roth) Harv.

Caulerpa fastigiata? Mont.

Codium decorticatum (Woodw.) Howe

\section{PHAEOPHYCEAE}

Ectocarpus sp.

Levringia sp.

Chnoospora pacifica J. Ag.

Colpomenia sinuosa (Roth) Derb. et Sol.

Padina Vickersiae Hoyt

Spatoglossum Schroederi (Mert.) J. Ag.

Sargassum cymosum C. Ag. var. stenophyllum (Mert.) Grun.

\section{RHODOPHYCEAE}

Erythrocladia subintegra Rosenv.

Erythrotrichia sp.

Goniotrichum sp.

Porphyra sp.

Gymnogongrus Griffithsiae (Turn.) Mart.

Gelidium sp.

Pterocladia capillacea (Gml.) Born. et Flaht.

(*) Conheeido do Uruguai (ef. 15 p. 141). 


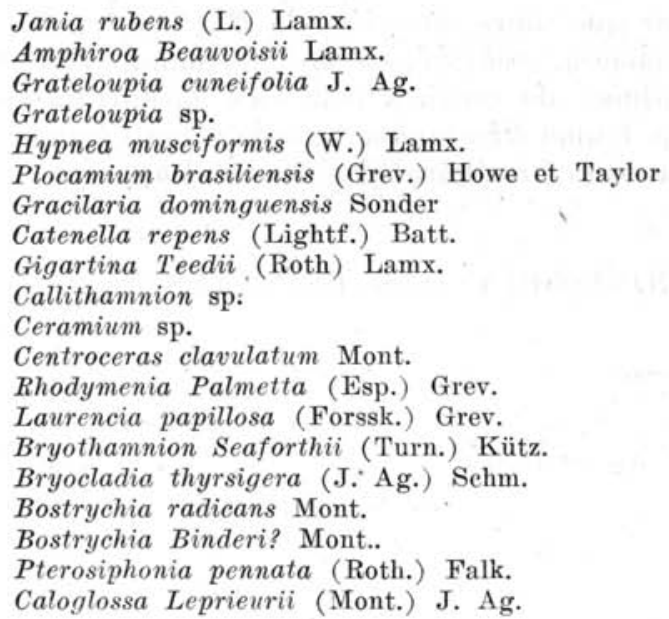

\section{RESUMO}

Esta nota é a primeira contribuição para a distribuição especial das algas marinhas do litoral paranaense (Caiobá).

São considerados três tipos de litoral:

a) Litoral rochoso sujeito à ação de arrebentação.

b) Litoral rochoso mais ou menos abrigado.

c) Manguesais.

Dentro do esquema proposto, o primeiro tipo é subdividido em 2 zonas :

$1^{\circ}{ }^{\circ}$ Zona dos borrifos, situada acima do limite médio da maré alta (fig. $2 \mathrm{n}^{\circ}$ 1), correspondente à "Splash zone" on à "Sprit zone" dos autores estrangeiros.

$2 .^{\circ}$ ) Zona de arrebentação (Ressaca), situada entre os níveis médios das marés baixa e alta, correspondendo à "Interdital zone" ou à "Gezeitenzone" dos autores estrangeiros. (fig. 2 n. ${ }^{0} 2$ ).

A primeira zona só conta com uma alga, Lyngbya confervoides e pelo menos mais duas espécies de moluseos do gênero Littorina.

A segunda zona, a mais rica tanto em algas como em animais, é essencialmente caracterizada em Caiobá pelas algas seguintes:

Levringia sp. Chaetomorpha media e Centroceras clavulatum na parte mais alta, associadas a balanoides (craca) e a Mytilus perna (mariseo). Mais abaixo domina Pterosiphonia pennata, Hypnea musciformis e Bryocladia thyrsigera. São apresentados também mais três tipos secundários dependendo das condiẹões locais dos rochedos. 
Esta sucessão de Littorina, Balanus e Mytilus é a mesma existente no sul da África, segundo se depreende dos trabalhos de Stephenson $(11,12)$ e de vários de seus colaboradores.

O segundo tipo apresenta também duas zonas, sendo a segunda a mais rica e variada, aparecendo aqui como dominante, uma associação na qual, Callithamnion, Laurencia papillosa, Gigartina Teedii e Sargassum cymosum são as mais abundantes.

É sugerida a existência de uma zona abaixo do limite inferior das marés baixas, zona essa representada por Rhodymenia Palmetta, Plocamium brasiliensis e Spatoglossum Schroederi pelo menos.

É feita uma rápida enumeração das algas dos manguesais.

\section{SUMMARY}

This paper is to be considered as a first contribution to the special distribution regarding marine sea-weed of the Parana coast (Caiobá).

We shall consider three coastal types:

a) Those of the rocky coast subject to the action of waves.

b) Those of the rocky coast but nevertheless more or less protected; and,

c) Those of the marshy ground.

Within the proposed plan, the firšt type is divided into two zones:

1) The Splash Zone located above the average limit of the tide (fig. 2 n. ${ }^{0}$ ). Foreign authors eall this zone also the Spritzone.

2) The Intertidal Zone (Ressaca), situated between the average of the levels of the tide. Foreign authors named this zone the Gezeitenzone (fig. 2 n. $^{0} 2$ ).

The first named of the two above zones contains only one species of sead-weed, Lyngbya confervoides and at least two species of molluks of the Littorina genus.

The second zone is the richer one of the two in animals as well as in sea-weed and is especially characterized in Caiobá by means of the following sea-weeds: Levringia sp., Chaetomorpha media and Centroceras clavulatum. These are to be found in the upper part together with balanoids (eraca) and Mytilus perna (mariseo).

Pterosiphonia pennata, Hypnea musciformis, and Bryocladia thyrsigera are prevalent in the lower part. Another three secondary types are to be found, however, this depending upon local conditions of the rocks. 
This succession of Littorina, balanoides and Mytilus is the same as that existing in the Southern part of Africa, as can be noted from works published by Stephenson $(11,12)$ and various others of his collaborators.

The second type also populates two zones, being the second one the richer and it also provides a grater variety. Here we find a group of sea-weeds and among them the following dominate Callithamnion, Laurencia papillosa, Gigartina Teedii and Sargassum cymosum.

It is believed that there exists another zone below the inferior limit of the tide and the same presents us the species Rhodymenia Palmetta, Plocamium brasiliensis and Spatoglossum Schroederi.

A quick enumeration of the sea-weeds living in the marshy ground is made.

\section{BIBLIOGRAFIA}

1. BIgARElla, João J. - Contribuição ao estudo da planície litorânea do Estado do Paraná. Arq. Biol. e Teenol. Sec. Ag. Ind. Com. Paraná I Art. 7: 75-111 Curitiba 1946.

2. CHAPMAN, V. J. - Marine Algal Ecology. Bot. Rev. 12 - 10 : 628-672, 1946.

3. DE TONI, J. B. - Sylloge Algarum 1899-1903.

4. ENGLER, A. U., PRANTL, K. - Die natürlichen Pflanzenfamilien I: 2 Leipzig, 1897.

5. FRITSCH, F. E. - The structure and reproduction of the Algae. I e III: Cambridge Univ. Press. Cambridge 1935 e 1945.

6. LUEDERWALT, H. - Os manguesais de Santos - sep. Rev. Mus. Paulista XI: 1-98. São Paulo 1919.

7. LUETzelburg, P. von - Estudo Botânico do Nordeste. Insp. Fed. Obras Contra as Sêcas. Minist. Viação e Ob. Publ. 57. Ser. I, A 3 vol. 1922-1923. Rio de Janeiro - Brasil.

8. OLTMANNS, F. - Morphologie u. Biologie der Algen vol. 3 - Jena 1923.

9. RAWITSCHER, Felix K. - Algumas noções sôbre a vegetação do litoral brasileiro. Bol. Assoe. Geogr. Bras. 5: 13-28. São Paulo 1944.

10. STELlfFeld, C. - Aspectos da zona da praia do Estado do Paraná (Brasil). Lilloa 20: 203-214. Tueuman 1949.

11. STEPHENSON, T. A. - The constitution of the inter-tidal fauna and flora of South Africa. I. - Journ. Linn. Soe. Zool. 40:487-536. 1939.

12. STEPHENSON, T. A. - Idem. II. Ann. Natal Mus. X, 3: 261-358. 1944.

13. TAYLOR, Wm. R. - Caribbean Marine Algae of the Allan Haneock Expedition 1939 Report n. ${ }^{\circ}$ 2. The Univ. South Cal. Press - Los Angeles, Cal. 1942.

14. TAYLOR, Wm. R. - A synopsis of the marine algae of Brasil. Rev. alg. V, 3-4, 279-313. Paris 1931.

15. TAYLOR, Wm. R. - Algae collected by the "Hassler", "Albatross" and Schmitt Expeditions II. Mich. Ac. Se. Arts and Let. XXIV, I: 127-164. 1938.

16. OLIVEIRA, Lejeune de - Levantamento Biogeográfico da Baía de Guanabara. Mem. Inst. Oswaldo Cruz 48: 363-391. 1950. 


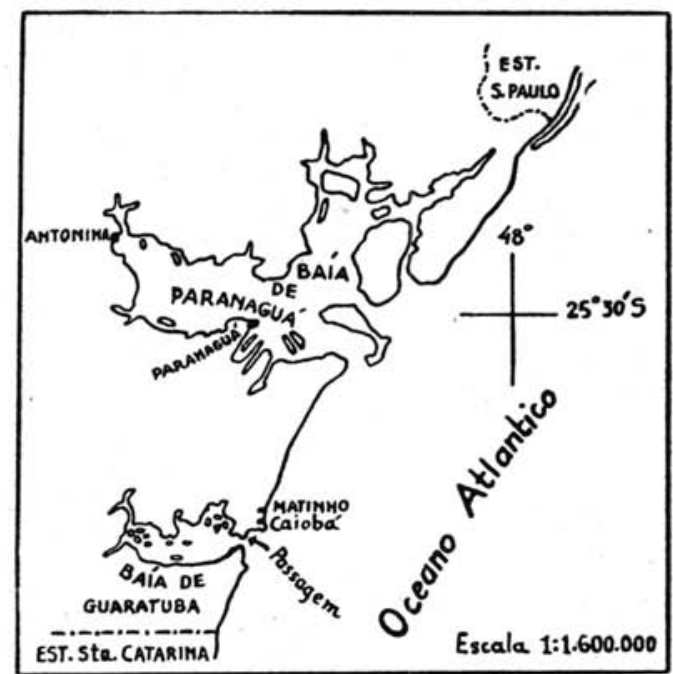

Fig. 1. - Mapa do litoral paranaense, seg. Bigarella (1) modificado. Assinalados os principais pontos de referência.

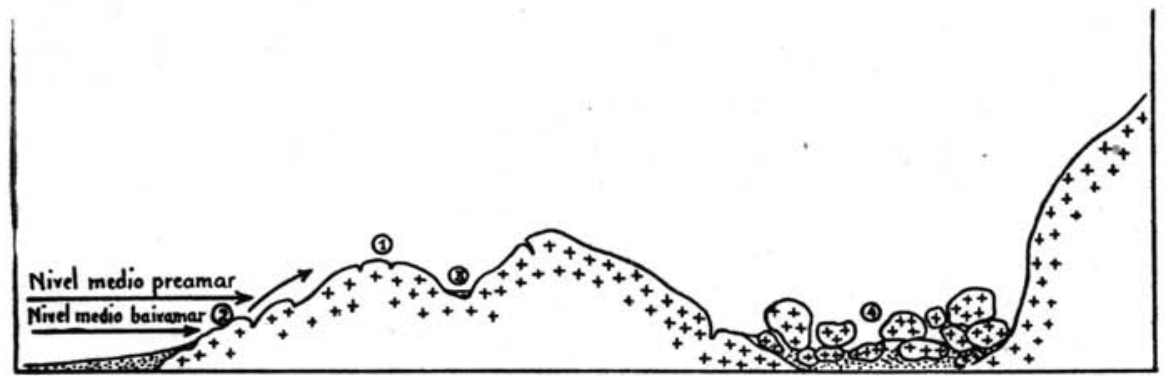

Fifg. 2. - Esquema da distribuiçăo de algas nos rochedos sujeitos à forte arrebentação. Caiobá. 4-11-1950. 


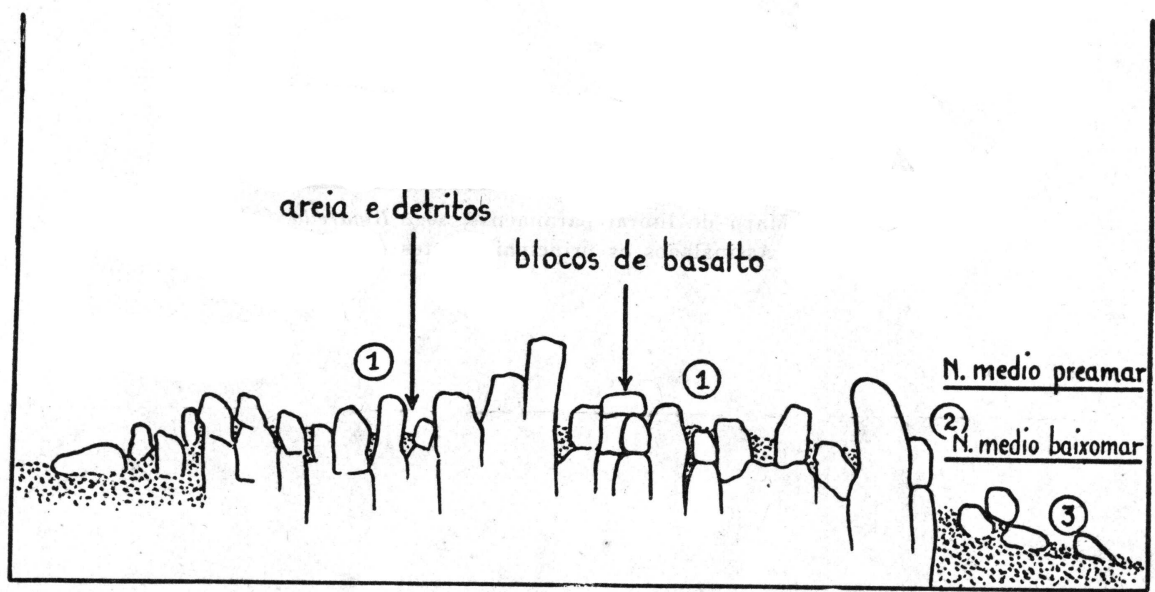

Fig. 3. - Esquema da distribuição de algas nos rochedos abrigados entre a Ilha do Farol e o Morro de Caiobá. 4-11-1950. 